\section{PROTEÓMICA Y BIOLOGÍA DE SISTEMAS PARA EL ESTUDIO DE LA ALERGIA ALIMENTARIA}

\author{
Mónica Carrera \\ Instituto de Investigaciones Marinas \\ Consejo Superior de Investigaciones Científicas \\ ORCID iD: https://orcid.org/0000-0003-2973-449X \\ mcarrera@iim.csic.es
}

Cómo citar este artículo/Citation: Carrera, M. (2020). Proteómica y biología de sistemas para el estudio de la alergia alimentaria. Arbor, 196 (795): a546. https://doi.org/10.3989/ arbor.2020.795n1010

Recibido: 26 febrero 2019. Aceptado: 29 octubre 2019.

RESUMEN: La alergia alimentaria es el cuarto principal problema de salud pública según datos de la Organización Mundial de la Salud (OMS). Afecta a un total de $6-8 \%$ de niños y a $2-4 \%$ de adultos. Debido a la actual gran prevalencia de la alergia alimentaria, se hace necesario el desarrollo de nuevos métodos de control, tratamiento y estudio. En esta revisión se presentan los enfoques proteómicos y de biología de sistemas más recientes para el estudio de la alergia alimentaria. En este sentido, se resumen con detalle las dos principales estrategias proteómicas (proteómica de descubrimiento y proteómica dirigida). También se describen los innovadores enfoques de biología de sistemas basados en datos proteómicos para el estudio de los mecanismos de la alergia alimentaria. Finalmente se presentan nuevas perspectivas y futuras direcciones.

PALABRAS CLAVE: proteómica; biología de sistemas; alimentos; alergia.

\section{PROTEOMICS AND SYSTEMS \\ BIOLOGY FOR THE STUDY OF FOOD ALLERGY}

Copyright: (C) 2020 CSIC. Este es un artículo de acceso abierto distribuido bajo los términos de la licencia de uso y distribución Creative Commons Reconocimiento 4.0 Internacional (CC BY 4.0).

ABSTRACT: Food allergy is the fourth most important public health problem according to the World Health Organization (WHO). A total of $6-8 \%$ of children and $2-4 \%$ of adults are affected. Currently, due to this high prevalence, fast and accurate detection methods for food allergy control, treatment and research are highly necessary. In this review, the most recent proteomics and systems biology approaches for the study of food allergy are presented. In this respect, two proteomics strategies (discovery and targeted proteomics) for the control and study of food allergy are summarized. Additionally, the innovative proteomics-based systems biology approaches for the study of the mechanisms of food allergy are described. Finally, new perspectives and future directions are described.

KEYWORDS: proteomics; systems biology; food; allergy. 


\section{INTRODUCCIÓN}

Las alergias alimentarias son uno de los principales problemas sanitarios que actualmente afectan a la mayor parte de los países industrializados. La Organización Mundial de la Alergia (WAO) destaca en su último informe la gran prevalencia que han experimentado estas enfermedades en los últimos veinte años, una tendencia que no muestra signos de moderación. Este considerable incremento está en estrecha correlación con la globalización de los mercados, con la mejora en la calidad de vida y con el control de las enfermedades infecciosas durante la infancia. Así, en la Unión Europea (UE), la alergia alimentaria es la enfermedad crónica más común en la edad infantil, llegando a tasas mayores del 6\% en algunos países. En España, la prevalencia de este tipo de enfermedades en la población general es de un 3-4\% (Sicherer y y Sampson, 2014).

Un total de catorce alimentos (pescado, crustáceos, moluscos, gluten, huevos, lácteos, cacahuetes, soja, apio, frutos de cáscara, mostaza, granos de sésamo y dióxido de azufre-sulfitos y altramuces) están declarados como alérgenos por la Autoridad Europea de Seguridad Alimentaria (European Food Safety Authority, EFSA).

En pacientes sensibilizados, los síntomas clínicos de la alergia alimentaria normalmente se manifiestan por medio de urticarias, conjuntivitis, rinitis, problemas gastrointestinales y dificultad respiratoria, dando lugar en el peor de los casos a un shock anafiláctico minutos después de la ingesta (Sampson, 2003).
Para garantizar la seguridad de los consumidores, la Comisión Europea en su Directiva 2007/68/EC obliga a los distintos países a declarar y asegurar la presencia de cualquier alérgeno o derivado del mismo en el etiquetado de todos los productos alimenticios. Asimismo, la autoridad de la EFSA reconoció la alta prevalencia y alto riesgo en todo el mundo y reclamó a la comunidad científica la necesidad de desarrollar nuevos métodos de control y detección de dichos alérgenos en los productos alimentarios.

La alergia alimentaria presenta un origen multifactorial en donde se incluyen factores genéticos, inmunológicos y ambientales (Lorente, Laffond, Dávila y Moreno, 2001). Es una hipersensibilidad alergénica tipo I mediada por la producción amplificada de inmunoglobulina $\mathrm{E}$ (IgE). Establecido el diagnóstico de alergia alimentaria, la exclusión del alimento alergénico, manteniendo los requerimientos nutricionales, es en la actualidad el único tratamiento apropiado para prevenir reacciones futuras. Hallar nuevas terapias para las alergias alimentarias, más allá de la simple supresión de la ingesta del alimento alergénico, forma parte de los objetivos actuales de la ciencia.

En este sentido, las poderosas metodologías -ómicas (Genómica, Proteómica, Metabolómica, etc) y de biología de sistemas están logrando un gran impacto en toda la comunidad científica. De hecho, a instancias de la comunidad científica dedicada al estudio de los alimentos, se ha establecido una nueva disciplina denominada Foodomics (Cifuentes, 2009; Herrero, Simó, García-Cañas, Ibánez y Cifuentes, 2012).

Figura 1. Principales áreas proteómicas y de biología de sistemas para el estudio de la alergia alimentaria

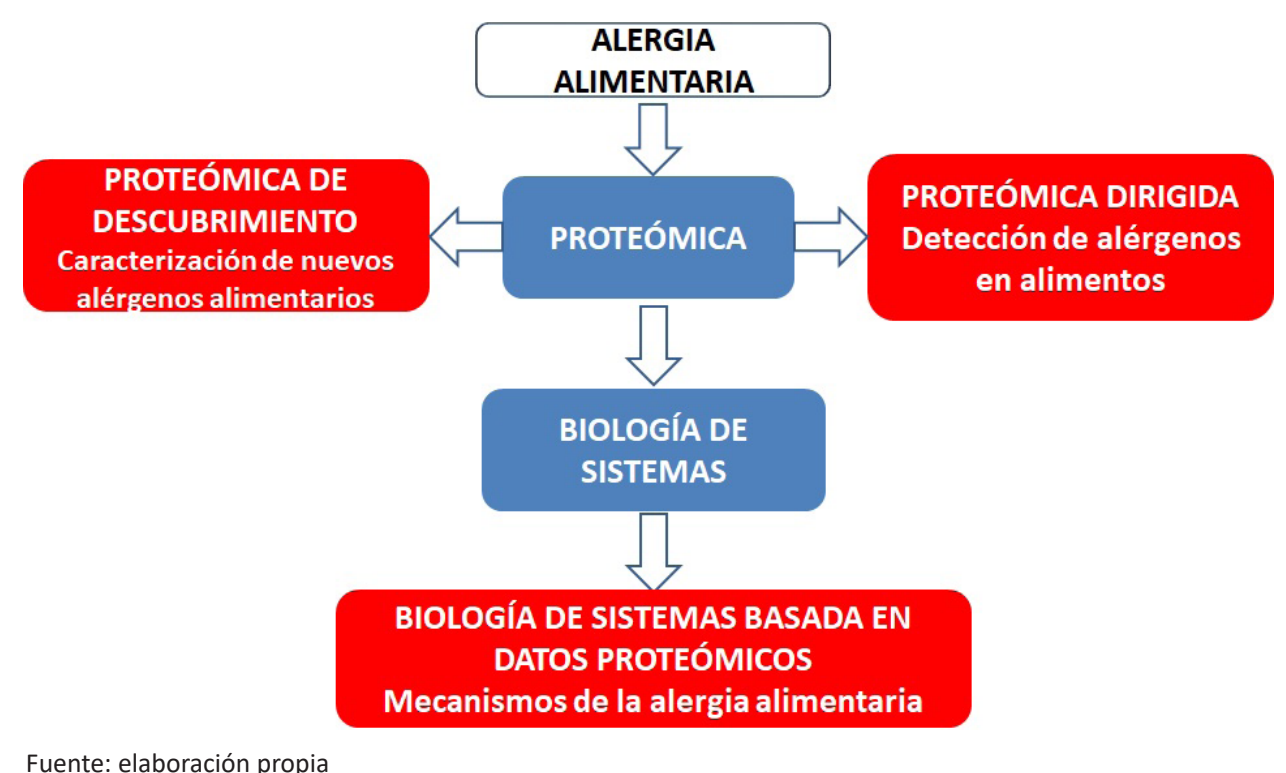


En esta revisión se describen los avances más recientes en las técnicas de proteómica de descubrimiento y dirigida (Discovery Proteomics y Targeted Proteomics), así como de las herramientas de biología de sistemas para el control y detección de alérgenos en los alimentos y para alcanzar una mayor comprensión de las bases moleculares de la alergia alimentaria (véase la figura 1). Resultados de gran transcendencia que están siendo útiles no solo para toda la comunidad científica sino también para las autoridades sanitarias e industrias alimentarias y farmacéuticas con el fin de garantizar la seguridad y proteger la salud de los consumidores.

\section{PROTEÓMICA DE DESCUBRIMIENTO EN LA ALERGIA ALIMENTARIA}

La proteómica de descubrimiento tiene como finalidad el estudio y caracterización a gran escala de los componentes de un determinado proteoma. Se basa en la identificación y cuantificación de las proteínas, la caracterización de sus modificaciones posttraduccionales (PTMs) y la identificación de péptidos y proteínas biomarcadoras. Así, las aproximaciones clásicas de la proteómica Bottom-up son prometedoras tecnologías para la caracterización e identificación de alérgenos alimentarios. Siguiendo esta metodología, la(s) proteína(s) de interés son sometidas a separación mediante electroforesis bidimensional (2-DE), electroforesis capilar (CE) o cromatografía líquida (LC), para a continuación ser digeridas utilizando enzimas (por ejemplo, tripsina) y los péptidos resultantes son entonces analizados por espectrometría de masas en tándem (MS/MS). Utilizando herramientas de búsqueda en las bases de datos, como SEQUEST, los espectros de fragmentación son asignados a posibles secuencias peptídicas y las asignaciones son seguidamente validadas utilizando programas como Percolator. Hasta la fecha, utilizando aproximaciones similares varias proteínas alergénicas han sido identificadas y caracterizadas (véase la tabla 1). Así, utilizando 2-DE, inmunobloting con sueros de pacientes alérgicos y análisis por espectrometría de masas con un analizador de tiempo de vuelo y desorción mediante láser asistida por matriz (MALDI-TOF) o por cromatografía líquida acoplada a espectrometría de masas en tándem (LC-MS/MS) fueron identificados nuevos alérgenos de crustáceos, sésamo, avellana, pistacho, fruta y gluten (Marzban, Herndl, Maghuly, Katinger y Laimer, 2008; Ortea, Cañas y Gallardo, 2009; Carrera, Cañas, Vázquez y Gallardo, 2010; Martínez-Esteso et al., 2016; Grishina, Bardina y Grishin, 2017).
Una novedosa estrategia desarrollada por nuestro grupo de investigación permitió la secuenciación de novo completa de nuevos alérgenos alimentarios (Carrera et al., 2010). Esta estrategia fue aplicada para la caracterización de todas las parvalbúminas beta ( $\beta$-PRVBs) de todas las especies comerciales de la familia Merlucciidae. Las $\beta$-PRVBs están consideradas como el principal alérgeno de los pescados (Elsayed y Bennich, 1975). Esta metodología se basa en la combinación de la estrategia clásica de la proteómica Bottom-up mediante geles 2-DE, la determinación de la masa molecular exacta de las proteínas intactas mediante espectrometría de masas de resonancia ciclotrónica de iones por transformada de Fourier (FTICRMS) y la monitorización de los péptidos teóricos necesarios para completar las secuencias faltantes mediante monitorización de la fragmentación de un ion seleccionado (SMIM) en un espectrómetro de masas con un analizador de trampa iónica lineal (LTQ). Así, con esta estrategia se logró la secuenciación de novo completa de veinticinco nuevas isoformas de $\beta$-PRVBs (Carrera et al., 2010).

\section{PROTEÓMICA DIRIGIDA EN LA ALERGIA ALIMENTARIA}

La proteómica dirigida es una estrategia que permite el análisis selectivo de un determinado número de proteínas o péptidos seleccionados que normalmente son considerados como biomarcadores. Para ello el analizador del espectrómetro de masas es programado para que se centre en el análisis de determinados compuestos de interés mediante monitorización de la reacción de un ion/múltiples seleccionado(s) (SRM/MRM) en analizadores tipo triple cuadrupolo (QqQ) o híbridos cuadrupolotrampa iónica (Q-Trap) o mediante SMIM (Jorge et al., 2007). Además, en equipos de espectrometría de masas de alta resolución (HRMS), como los espectrómetros de masas tipo Q-Orbitrap, se logra una mejora en la velocidad, sensibilidad y rango dinámico de la monitorización, denominándose en este caso monitorización de la reacción en paralelo de un ion/ múltiples seleccionado(s) (PRM). Estos últimos modos de trabajo (SMIM/PRM) son los más avanzados y presentan dos importantes ventajas frente a los otros modos de barrido: por un lado, consiguen un considerable incremento en la sensibilidad debido al promedio de varios espectros de fragmentación (MS/MS), de principal importancia para confirmar la naturaleza o secuencia de la especie identificada, y por el otro, es un modo de trabajo que permite obtener cromatogramas virtuales para los diferentes iones fragmento. 
En el caso de la alergia alimentaria, la proteómica dirigida se ha aplicado para la detección y cuantificación en los productos alimenticios de la presencia o no de determinados alérgenos alimentarios (véase la tabla 1). Así, la monitorización mediante LC-SRM/MRM en instrumentos tipo QqQ o Q-Trap ha permitido la detección y cuantificación específica de la presencia de gluten, huevos, lácteos, soja, avellanas, nueces, almendras o cacahuetes en productos alimenticios (Pilolli, Angelis y Monaci, 2017; Angelis, Pilolli y Monaci, 2017; Ansari, Stoppacher, Rudolf, Schuhmacher y Baumgartner, 2011; Careri, Elviri, Lagos et al., 2008; Cryar et al., 2012; Heick, Fischer y Pöpping, 2011; Lutter, Parisod y Weymuth, 2011; Martínez-Esteso et al., 2016; Pedreschi, Nørgaard y Maquet, 2012; Sealey-Voyksner, Khosla, Voyksner y Jorgenson, 2010) (véase la tabla 1). Asimismo, utilizando instrumentos de alta resolución como el Q-Orbitrap fue posible la detección a nivel de trazas de alérgenos de cacahuetes, leche o huevos en otros productos alimenticios (Monaci, Angelis, Bavaro y Pilolli, 2015; Monaci, Losito, Angelis, Pilolli y Visconti, 2013; Monaci, Losito, Palmisano y Visconti, 2011). Por otra parte, hay que destacar que nuestro grupo de investigación desarrolló una novedosa metodología para la detección de todas las $\beta$-PRVBs de pescado en cualquier producto alimenticio (Carrera, Cañas y Gallardo, 2012). El método se basa en los siguientes pasos: (i) purificación rápida de las $\beta$-PRVBs (proteínas termoestables) por tratamiento con calor (tiempo: 45 min.), (ii) digestión ultrarrápida con tripsina empleando ultrasonidos de alta intensidad focalizados (HIFU) (tiempo: 2 min.), y (iii) monitorización mediante SMIM de los péptidos biomarcadores para la detección de las $\beta$-PRVBs en un LTQ (tiempo: $60 \mathrm{~min}$.). Esta estrategia permite la detección directa del principal alérgeno de los pescados ( $\beta$-PRVBs) en cualquier producto alimenticio en menos de dos horas. Es el método más rápido descrito hasta el momento. Esta estrategia también se ha aplicado para la detección de la proteína alergénica y termoestable Ani s 9, específica de los Anisákidos, en cualquier producto alimenticio (Carrera, Gallardo, Pascual, González y Medina, 2016).

Por otra parte, los espectrómetros de masas en modo de adquisición independiente de datos (DIA) en equipos de HRMS o en equipos de movilidad iónica (DIA-IM-MS) han permitido la detección de trazas de huevo, leche o cacahuetes en varios alimentos (Johnson et al., 2016; Monaci et al., 2013). Finalmente, los métodos de proteómica Top-Down permiten la detección y cuantificación de los alérgenos alimentarios a nivel de proteína intacta sin necesidad de digestión previa con enzimas. Para ello, se necesitan de equipos HRMS como el FTICR-MS o el Orbitrap Fusion implementada con fotodisociación inducida por ultravioleta UVPD. Estos métodos se han aplicado para la detección de leche en muestras de zumos de frutas (Monaci y van Hengel, 2008) y para la detección de $\beta$-PRVBs en pescado (Carrera et al., 2015).

\section{BIOLOGÍA DE SISTEMAS EN LA ALERGIA ALIMENTARIA}

La biología de sistemas es una ciencia integradora que utiliza la información obtenida de las ciencias -ómicas, como la proteómica, junto con las herramientas bioinformáticas y la modelización computacional para entender y predecir las propiedades de los sistemas biológicos desde un punto de vista holístico.

Aunque la respuesta de los linfocitos $\mathrm{T}$ y $\mathrm{B}$ a un alérgeno presenta un proceso similar a la respuesta frente a un antígeno convencional, es decir, el linfocito T (del tipo Th2) reconoce un péptido del alérgeno procesado y presentado por una célula presentadora de antígeno en la molécula del complejo mayor de histocompatibilidad ( $\mathrm{MHC}$ ) de clase II, lo que desemboca en la cooperación y diferenciación de los linfocitos $\mathrm{B}$ para dar lugar a células plasmáticas secretoras de IgE, parece ser que su inapropiado mecanismo de activación presenta un papel fundamental en la etapa de sensibilización de los pacientes atópicos (Maggi, 1998). Por esta razón, la investigación de los mecanismos moleculares de esta inapropiada activación de las células $T$ y $B$ es de gran interés científico, no solamente para comprender mejor los procesos alergénicos sino también para entender todos aquellos procesos de respuesta inapropiada de los linfocitos, en donde se interrumpe la tolerancia inmunológica. Así las novedosas técnicas de biología de sistemas permitirán desarrollar modelos computacionales de los mecanismos de transducción de señales y de activación de los linfocitos $T$ y $B$ en los casos de alergia alimentaria. Dichos modelos podrían identificar potenciales dianas diagnósticas y terapéuticas. En vista de esto, nuestro grupo está trabajando en herramientas de biología de sistemas utilizando datos proteómicos para alcanzar una mayor comprensión de las bases moleculares de la alergia al pescado. Para ello, se están desarrollando estudios de activación de las células T en casos de alergia al pescado, involucrando principalmente cambios en la abundancia proteica, interacciones proteína-proteína y modificaciones post-traduccionales (PTMs), principalmente fosforilaciones (Carrera, Cañas. y López-Ferrer, 2017). Así la aplicación de estas herramientas de biología de sistemas y simulación matemática culmina- 
rán con la generación de un modelo in-silico que prediga el comportamiento de las células T en el caso de la alergia al pescado. Este modelo tendrá un impacto directo en la identificación de potenciales dianas para el desarrollo de nuevos fármacos que logren el tratamiento y control de estas enfermedades alérgicas.

Tabla 1. Resumen de las últimas aplicaciones proteómicas para el estudio de la alergia alimentaria

\begin{tabular}{|c|c|c|c|}
\hline Proteómica & Alérgeno alimentario & Herramientas Proteómicas & Referencias \\
\hline \multirow[t]{5}{*}{ Descubrimiento } & Frutas & 2-DE, immunoblot & Marzban et al., 2008 \\
\hline & $\begin{array}{l}\text { Crustáceos, sésamo, } \\
\text { avellanas, pistachos }\end{array}$ & 2-DE, inmunoblot & Grishina et al., 2017 \\
\hline & Gluten & LysC, tripsina, quimiotripsina, LC-MS/MS DDA & Martínez-Esteso et al., 2016 \\
\hline & $\begin{array}{l}\text { Crustáceos (Arginina } \\
\text { quinasa) }\end{array}$ & $\begin{array}{l}\text { Proteómica Bottom-up, tripsina, LC-MS/MS } \\
\text { DDA }\end{array}$ & Ortea et al., 2009 \\
\hline & $\begin{array}{l}\text { Pescado (Parvalbúmi- } \\
\text { nas, } \beta \text {-PRVBs) }\end{array}$ & $\begin{array}{l}\text { Proteómica Bottom-up, tripsina, Glu-C, LC- } \\
\text { MS/MS DDA, FTICR-MS, SMIM }\end{array}$ & Carrera et al., 2010 \\
\hline \multirow[t]{22}{*}{ Dirigida } & Gluten & LC-SRM & Martínez-Esteso et al., 2016 \\
\hline & Huevos, leche & LC-SRM & Angelis et al., 2017 \\
\hline & $\begin{array}{l}\text { Huevos, leche, soja, } \\
\text { avellanas, cacahuetes }\end{array}$ & LC-SRM & Pilolli et al., 2017 \\
\hline & Gluten & LC-MRM & $\begin{array}{l}\text { Sealey-Voyksner et al., } \\
2010\end{array}$ \\
\hline & Leche & LC-MRM & Ansari et al., 2011 \\
\hline & $\begin{array}{l}\text { Leche, huevos, soja, } \\
\text { cacahuetes, avellanas, } \\
\text { nueces, almendras }\end{array}$ & LC-MRM & Heick et al, 2011 \\
\hline & Leche & LC-MRM & Lutter et al., 2011 \\
\hline & Huevos & LC-MRM & Cryar et al, 2012 \\
\hline & Cacahuetes & LC-MRM & Pedreschi et al., 2012 \\
\hline & Pescado & LC-MRM & Sun et al., 2019 \\
\hline & Nueces & LC-MRM $^{3}$ & Korte y Brockmeyer, 2016 \\
\hline & Cacahuetes & Microfluídica-MRM & Sayers et al., 2018 \\
\hline & Cacahuetes & ICP-MS, LC-MRM & $\begin{array}{l}\text { Careri, Elviri, Maffini et al., } \\
2008\end{array}$ \\
\hline & Gluten & LC-MS/MS DDA & $\begin{array}{l}\text { Weber, Chantal y Godefroy, } \\
2009\end{array}$ \\
\hline & Huevos & LC-MS/MS DDA & Lee y Kim, 2010 \\
\hline & Leche & LC-MS/MS DDA & Hong et al., 2012 \\
\hline & Leche & LC-MS/MS DDA & $\begin{array}{l}\text { Cereda, Kravchuk, D'Amato, } \\
\text { Bachi y Righetti, } 2010\end{array}$ \\
\hline & Leche & LC-MS/MS DDA & $\begin{array}{l}\text { D’Amato, Kravchuk, Bachi y } \\
\text { Righetti, } 2010\end{array}$ \\
\hline & Leche & LC-MS/MS DDA (XIC) & $\begin{array}{l}\text { Monaci, Losito, Palmisano y } \\
\text { Visconti, } 2010\end{array}$ \\
\hline & Cacahuetes & LC-MS/MS DDA (XIC) & $\begin{array}{l}\text { Hebling, McFarland, Calla- } \\
\text { han y Ross, } 2013\end{array}$ \\
\hline & Cacahuetes & LC-HR-MS/MS DDA (XIC) & Monaci et al., 2015 \\
\hline & Lupino & LC-MS/MS DDA, LC-SRM & $\begin{array}{l}\text { Mattarozzi, Bignardi, Elviri y } \\
\text { Careri, } 2012\end{array}$ \\
\hline
\end{tabular}




\begin{tabular}{|c|c|c|c|}
\hline Proteómica & Alérgeno alimentario & Herramientas Proteómicas & Referencias \\
\hline & Leche & LC-HR-MS/MS (XIC, SMIM) & Monaci et al., 2011 \\
\hline & Cacahuetes & LC-MS/MS SMIM & $\begin{array}{l}\text { Careri, Elviri, Lagos et al, } \\
2008\end{array}$ \\
\hline & Crustáceos & LC-MS/MS SMIM & $\begin{array}{l}\text { Ortea, Cañas y Gallardo, } \\
2011\end{array}$ \\
\hline & Pescado ( $\beta$-PRVBs) & HIFU-LC-MS/MS SMIM & Carrera et al., 2011 \\
\hline & Pescado ( $\beta$-PRVBs) & HIFU-LC-MS/MS SMIM & Carrera et al., 2012 \\
\hline & Anisákidos (Anis s 9) & HIFU-LC-MS/MS PRM & Carrera et al., 2016 \\
\hline & Huevos, Leche & LC-HR-MS/MS DIA (XIC) & Monaci et al., 2013 \\
\hline & Cacahuetes & LC-DIA-IM-MS & Johnson et al., 2016 \\
\hline & Leche & Proteómica Top-Down, LC-MS SIM & Monaci et al., 2008 \\
\hline & Pescado ( $\beta$-PRVBs) & Proteómica Top-Down, HRMS-UVPD & Carrera et al., 2015 \\
\hline
\end{tabular}

2-DE, electroforesis bidimensional; MALDI-TOF MS, espectrometría de masas con analizador de tiempo de vuelo y desorción mediante láser asistida por matriz; FTICR-MS, espectrometría de masas de resonancia ciclotrónica de iones por transformada de Fourier; LC-MS/MS, cromatografía líquida acoplada a espectrometría de masas en tándem; HR-MS/MS, espectrometría de masas en tándem de alta resolución; SIM, monitorización de un ion seleccionado; SMIM, monitorización de la fragmentación de un ion seleccionado; SRM, monitorización de la reacción de un ion seleccionado; MRM, monitorización de la reacción de múltiples iones seleccionados; MRM $^{3}$, monitorización de la reacción de múltiples iones seleccionados al cubo; PRM, monitorización de la reacción en paralelo de un ion seleccionado; DDA, adquisición dependiente de datos; DIA, adquisición independiente de datos; XIC, cromatograma de un ion extraído; HIFU, ultrasonidos de alta intensidad focalizados; ICP-MS, espectrometría de masas por plasma acoplado inductivamente; IM-MS, espectrometría de masas de movilidad iónica; HRMS, espectrometría de masas de alta resolución; UVPD, fotodisociación por ultravioleta.

Fuente: elaboración propia.

\section{CONCLUSIONES Y NUEVAS PERSPECTIVAS}

En esta revisión se resumen las capacidades de la proteómica y de la biología de sistemas para el estudio de la alergia alimentaria. La combinación de las dos principales estrategias proteómicas (Descubrimiento y Dirigida) han permitido la secuenciación de novo completa de nuevos alérgenos alimentarios. Asimismo, la monitorización de varios péptidos biomarcadores de alérgenos mediante SMIM o PRM permite la detección rápida de los alérgenos en cualquier producto alimenticio en menos de 2 horas.

Por otra parte, la aplicación de la cuantificación absoluta mediante AQUA-LC-MRM (Ahsan, Rao, Gruppuso, Ramratnam y Salomon, 2016), el uso de la electroforesis capilar (CE) acoplada a aproximaciones de proteómica Top-Down en instrumentos de HRMS (Álvarez, Montero, Llorens, Castro-Puyana y Cifuentes, 2018), la proteómica Cross-Talk y el empleo de los nuevos modos de fragmentación como HCD (high-collision dissociation), ETDhcD (electron-transfer-high-collision dissociation) y UVPD (ultraviolet photo-dissociation) para lograr la secuenciación de novo completa de proteínas intactas, son las nuevas perspectivas que se están empezando a utilizar en el campo de la alergia alimentaria. Además, la incorporación de estos resultados en sistemas portátiles, nano-biosensores tipo lab-on-a-chip, arrays de proteínas o sistemas de microfluídica (Neethirajan, Weng, Tah, Cordero y Ragavan, 2018), ofrecen nuevas ventajas para la industria alimentaria y las autoridades de control alimentario para poder realizar los análisis de control de los alérgenos alimentarios en sus propias compañías sin necesidad de personal especializado e instrumentación costosa con el fin de garantizar al consumidor una seguridad alimentaria.

\section{AGRADECIMIENTOS}

La revisión presentada tiene su origen en la actividad científica de la autora y de los coautores que figuran en sus publicaciones, gracias a la financiación de los proyectos de investigación de la Fundación Ramón Areces (XVII Concurso Nacional) y de la Agencia Gallega de Innovación GAIN, Xunta de Galicia, Proyectos de Excelencia 2018 (IN607D 2017/01). 


\section{BIBLIOGRAFÍA}

Ahsan, N., Rao, R. S. P., Gruppuso, P. A., Ramratnam, B. y Salomon, A. R. (2016). Targeted proteomics: current status and future perspectives for quantification of food allergens. Journal of Proteomics, 143, pp. 15- 23. https://doi. org/10.1016/j.jprot.2016.04.018

Álvarez, G., Montero, L., Llorens, L., Castro-Puyana, M. y Cifuentes, A. (2018). Recent advances in the application of capillary electromigration methods for food analysis and Foodomics. Electrophoresis, 39, pp. 136-159. https://doi. org/10.1002/elps.201700321

Angelis, E. de, Pilolli, R. y Monaci, L. (2017). Coupling SPE on-line pre-enrichment with HPLC and MS/MS for the sensitive detection of multiple allergens in wine. Food Control, 73, pp. 814820. https://doi.org/10.1016/j.foodcont.2016.09.031

Ansari, P., Stoppacher, N., Rudolf, J., Schuhmacher, R. y Baumgartner, S. (2011). Selection of possible marker peptides for the detection of major ruminant milk proteins in food by liquid chromatography-tandem mass spectrometry. Analytical and Bioanalytical Chemistry, 399, pp. 1105-1115. https://doi. org/10.1007/s00216-010-4422-0

Careri, M., Elviri, L., Lagos, J. B., Mangia, A. Speroni, F. y Terenghi, M. (2008). Selective and rapid immunomagnetic beadbased sample treatment for the liquid chromatography-electrospray ion-trap mass spectrometry detection of Ara h3/4 peanut protein in foods. Journal of Chromatogr A, 1206, pp. 89-94. https:// doi.org/10.1016/j.chroma.2008.07.091

Careri, M., Elviri, L., Maffini, M., Mangia, A., Mucchino, C. y Terenghi, M. (2008). Determination of peanut allergens in cereal-chocolate-based snacks: metaltag inductively coupled plasma mass spectrometry immunoassay versus liquid chromatography/ electrospray ionization tandem mass spectrometry. Rapid Communication in Mass Spectrometry, 22, pp. 807-811. https://doi. org/10.1002/rcm.3427

Carrera, M., Cañas, B. y Gallardo, J. M (2012). Rapid direct detection of the major fish allergen, parvalbumin, by selected $\mathrm{MS} / \mathrm{MS}$ ion monitoring mass spectrometry. Journal of Proteomics, 75, pp. 3211- 3220. https://doi. org/10.1016/j.jprot.2012.03.030
Carrera, M., Cañas, B. y López-Ferrer, D. (2017). Fast global phosphoproteome profiling of Jurkat T cells by HIFUTiO2- SCX-LC-MS/MS. Analytical Chemistry, 89, pp. 8853-8862. https://doi. org/10.1021/acs.analchem.7b01321

Carrera, M., Cañas, B., Lopez-Ferrer, D., Piñeiro, C., Vázquez, J. y Gallardo, J. M. (2011). Fast monitoring of speciesspecific peptide biomarkers using highintensity-focused-ultrasound-assisted tryptic digestion and selected MS/ MS ion monitoring. Analytical Chemistry, 83, pp. 5688-5695. https://doi. org/10.1021/ac200890w

Carrera, M., Cañas, B., Vázquez, J. y Gallardo, J. M. (2010). Extensive de novo sequencing of new parvalbumin isoforms using a novel combination of bottom-up proteomics, accurate molecular mass measurement by FTICR-MS, and selected MS/MS ion monitoring. Journal of Proteome Research, 9, pp. 4393-4406. https://doi.org/10.1021/pr100163e

Carrera, M., Gallardo, J. M., Pascual, S., González, A. F. y Medina, I. (2016). Protein biomarker Discovery and fast monitoring for the identification and detection of Anisakids by parallel reaction monitoring (PRM) mass spectrometry. Journal of Proteomics, 142, pp. 130-137. https:// doi.org/10.1016/j.jprot.2016.05.012

Carrera, M., Weisbrod, C., Lopez-Ferrer, D., Huguet, R., Gallardo, J. M., Schwartz, J. y Huhmer, A. (2015). Top-down, highthroughput of thermostable allergens using complementary MS/MS fragmentation strategies. Thermo Fisher Scientific. PN64488-EN 0615S.

Cereda, A., Kravchuk, A. V., D’Amato, A., Bachi, A. y Righetti, P. G. (2010). Proteomics of wine additives: mining for the invisible via combinatorial peptide ligand libraries. Journal of Proteomics, 73, pp. 1732-1739. https://doi. org/10.1016/j.jprot.2010.05.010

Cifuentes, A. (2009). Food analysis and foodomics. Journal of Chromatography A, 1216, pp. 7109-7110. https://doi. org/10.1016/j.chroma.2009.09.018

Cryar, A., Pritchard, C., Burkitt, W., Walker, M., O'Connor, G. y Quaglia, M. (2012). A mass spectrometry-based reference method for the analysis of lysozyme in wine and the production of certified reference materials. Journal of the Association of Public Analysis, 40, pp. 77-80.
D’Amato, A., Kravchuk, A. V., Bachi, A. y Righetti, P. G. (2010). Noah's nectar: the proteome content of a glass of red wine. Journal of Proteomics, 73, pp. 2370-2377. https://doi.org/10.1016/j. jprot.2010.08.010

Elsayed, S. y Bennich, H. (1975). The primary structure of allergen $M$ from cod. Scandinavian Journal of $\mathrm{Im}$ munology, 4, pp. 203-208. https:// doi.org/10.1111/j.1365-3083.1975. tb02618.x

Grishina, G., Bardina, L. y Grishin, A. (2017). 2D-electrophoresis and immunoblotting in food allergy. Methods in Molecular Biology, 1592, pp. 59-69. https://doi. org/10.1007/978-1-4939-6925-8_5

Hebling, C. M., McFarland, M. A., Callahan, J. H. y Ross, M. M. (2013). Global proteomic screening of protein allergens and advanced glycation endproducts in thermally processed peanuts. Journal of Agricultural and Food Chemistry, 61, pp. 5638-5648. https://doi.org/10.1021/ jf303554t

Heick, J., Fischer, M. y Pöpping, B. (2011). First screening method for the simultaneous detection of seven allergens by liquid chromatography mass spectrometry. Journal of Chromatography A, 1218, pp. 938-943. https://doi.org/10.1016/j. chroma.2010.12.067

Herrero, M., Simó, C., García-Cañas, V., Ibánez, E. y Cifuentes, A. (2012). Foodomics: MS-based strategies in modern food science and nutrition. Mass Spectrometry Reviews, 31, pp. 49-69. https://doi.org/10.1002/mas.20335

Hong, C., Jiang, H., Lü, E., Wu, Y., Guo, L., Xie, Y., Wang, C. y Yang, Y. (2012). Identification of milk component in ancient food residue by proteomics. PLOS One, 7, e37053. https://doi.org/10.1371/ journal.pone.0037053

Johnson, P. E., Sayers, R. L., Gethings, L. A., Balasundaram, A., Marsh, J. T., Langridge, J. I., Clare Mills, E. N. (2016). Quantitative proteomic profiling of peanut allergens in food ingredients used for oral food challenges. Analytical Chemistry, 88, pp. 5689-5695. https://doi. org/10.1021/acs.analchem.5b04466

Jorge, I., Casas, E. M., Villar, M., OrtegaPérez, I., López-Ferrer, D., MartínezRuíz, A. [...] y Vázquez, J. (2007). Highsensitivity analysis of specific peptides 
in complex samples by selected MS/ MS ion monitoring and linear ion trap mass spectrometry: application to biological studies. Journal of Mass Spectrometry, 42, pp. 1391-1403. https://doi. org/10.1002/jms.1314

Korte, R. y Brockmeyer, J. (2016). MRM3based LC-MS multi-method for the detection and quantification of nut allergens. Analytical and Bioanalytical Chemistry, 408, pp. 7845-7855. https://doi. org/10.1007/s00216-016-9888-y

Lee, J. Y. y Kim, C. J. (2010). Determination of allergenic egg proteins in food by protein-, mass spectrometry-, and DNAbased methods. Journal of AOAC International, 93, pp. 462-477.

Lorente, F., Laffond, E., Dávila, I. y Moreno, E. (2001). Mecanismos de tolerancia inmunológica. Prevención primaria de la alergia a alimentos. Alergología e Inmunología Clínica,16, pp. 58-75.

Lutter, P., Parisod, V. y Weymuth, H. (2011). Development and validation of a method for the quantification of milk proteins in food products based on liquid chromatography with mass spectrometric detection. Jour nal of AOAC International, 94, pp. 1043-1059. https://doi.org/10.1093/ jaoac/94.4.1043

Maggi, E. (1998). The TH1/TH2 paradigm in allergy. Immunotechnology, 3, pp. 233244. https://doi.org/10.1016/S13802933(97)10005-7

Martínez-Esteso, M. J., Nørgaard, J., Brohée, M., Haraszi, R., Maquet, A. y O'Connor, G. (2016). Defining the wheat gluten peptide fingerprint via a discovery and targeted proteomics approach. Journal of Proteomics, 147, pp. 156-168. https://doi.org/10.1016/j. jprot.2016.03.015

Marzban, G., Herndl, A., Maghuly, F., Katinger, H. y Laimer, M. (2008). Mapping of fruit allergens by 2D electrophoresis and immunodetection. Expert Reviews in Proteomics, 5, pp. 61-75. https://doi. org/10.1586/14789450.5.1.61

Mattarozzi, M., Bignardi, C., Elviri, L. y Careri, M. (2012). Rapid shotgun proteomic liquid chromatography-electrospray ionization-tandem mass spectrometrybased method for the lupin (Lupinus albus L.) multi-allergen determination in foods. Journal of Agricultural and Food Chemistry, 60, pp. 5841-5846. https:// doi.org/10.1021/jf302105r
Monaci, L., Angelis, E. de, Bavaro, S. L. y Pilolli, R. (2015). High-resolution Orbitrap ${ }^{\mathrm{TM}}$-based mass spectrometry for rapid detection of peanuts in nuts. Food Additive \& Contaminants: Part A: Chemistry, Analysis, Control, Exposure \& Risk Assessment, 32, pp. 1607-1616. https://doi.org/10.1080/19440049.201 5.1070235

Monaci, L. y van Hengel, A. J. (2008). Development of a method for the quantification of whey allergen traces in mixedfruit juices based on LC with MS detection. Journal of Chromatography, 1192 pp. 113-120. https://doi.org/10.1016/j. chroma.2008.03.041

Monaci, L., Losito, I., Angelis, E. de, Pilolli, R. y Visconti, A. (2013). Multi-allergen quantification of fining-related egg and milk proteins in white wines by highresolution mass spectrometry. Rapid Communication in Mass Spectrometry, 27, pp. 2009-2018. https://doi. org/10.1002/rcm.6662

Monaci, L., Losito, I., Palmisano, F. y Visconti, A. (2010). Identification of allergenic milk proteins markers in fined White wines by capillary liquid chromatographyelectrospray ionization-tandem mass spectrometry. Journal of Chromatography $A, 1217$, pp. 4300- 4305. https:// doi.org/10.1016/j.chroma.2010.04.035

Monaci, L., Losito, I., Palmisano, F. y Visconti, A. (2011). Reliable detection of milk allergens in food using a high-resolution, stand-alone mass spectrometer. Journal of AOAC International, 94, pp. 1034-1042. https://doi.org/10.1093/ jaoac/94.4.1034

Neethirajan, S., Weng, X., Tah, A., Cordero, O. y Ragavan, K. V. (2018). Nano-biosensor platforms for detecting food allergens - New trends. Sensing and BioSensing Research, 18, pp. 13-30. https:// doi.org/10.1016/j.sbsr.2018.02.005

Ortea, I., Cañas, B. y Gallardo, J. M. (2009). Mass spectrometry characterization of species-specific peptides from arginine kinase for the identification of commercially relevant shrimp species. Journal of Proteome Research, 8, pp. 5356-5362. https://doi.org/10.1021/pr900663d

Ortea, I., Cañas, B. y Gallardo, J. M. (2011) Selected tandem mass spectrometry ion monitoring for the fast identification of seafood species. Journal of Chromatography $A, 1218$, pp. 4445-4451. https:// doi.org/10.1016/j.chroma.2011.05.032
Pedreschi, R., Nørgaard, J. y Maquet, A. (2012). Current challenges in detecting food allergens by shotgun and targeted proteomic approaches: a case study on traces of peanut allergens in baked cookies. Nutrients, 4, pp. 132-150. https:// doi.org/10.3390/nu4020132

Pilolli, R., Angelis, E. de y Monaci, L. (2017). Streamlining the analytical workflow for multiplex MS/MS allergen detection in processed foods. Food Chemistry, 221, pp. 1747-1753. https://doi. org/10.1016/j.foodchem.2016.10.110

Sampson, H. A. (2003). Anaphylaxis and emergency treatment. Pediatrics, 111, pp. 1601-1608.

Sayers, R. L., Gethings, L. A., Lee, V., Balasundaram, A., Johnson, P. E., Marsh, J. A., Wallace, A., Brown, H., Rogers, A., Langridge, J. I., Mills, E. N. C. (2018). Microfluidic separation coupled to mass spectrometry for quantification of peanut allergens in a complex food matrix. Journal of Proteome Research, 17, pp. 647-655. https://doi.org/10.1021/acs. jproteome.7b00714

Sealey-Voyksner, J. A., Khosla, C., Voyksner, R. D. y Jorgenson, J. W. (2010). Novel aspects of quantitation of immunogenic wheat gluten peptides by liquid chromatography-mass spectrometry/mass spectrometry. Journal of Chromatography $A, 1217$, pp. 4167-4183. https://doi. org/10.1016/j.chroma.2010.01.067

Sicherer, S. H. y Sampson, H. A. (2014). Food allergy: epidemiology, pathogenesis, diagnosis and treatment. Journal of Allergy and Clinical Immunology, 133, pp. 291-307. https://doi.org/10.1016/j. jaci.2013.11.020

Sun, L., Lin, H., Li, Z., Sun, W., Wang, J., Wu, H., Ge, M., Ahmed, I. y Pavase, T. R. (2019). Development of a method for the quantification of fish major allergen parvalbumin in food matrix via liquid chromatography-tandem mass spectrometry with multiple reaction monitoring. Food Chemistry 276, pp. 358-365. https://doi.org/10.1016/j.foodchem.2018.10.014

Weber, D., Chantal, C. y Godefroy, S. B. (2009). Emerging analytical methods to determine gluten markers in processed food-method development in support of standard setting. Analytical and Bioanalytical Chemistry, 395, pp. 111-117. https://doi.org/10.1007/s00216-0092943-1 